\title{
Fixed time synchronization of delayed quaternion-valued memristor-based neural networks
}

\author{
Dingyuan Chen ${ }^{1}$, Weiwei Zhang ${ }^{1,2^{*}}$ (D) Jinde $\mathrm{CaO}^{2}$ and Chuangxia Huang ${ }^{3}$
}

\author{
"Correspondence: \\ wwzhahu@aliyun.com \\ 'School of Mathematics and \\ Physics, Anqing Normal University, \\ Anqing, China \\ ${ }^{2}$ School of Mathematics, Southeast \\ University, Nanjing, China \\ Full list of author information is \\ available at the end of the article
}

\begin{abstract}
This paper investigates the fixed time synchronization issue for a class of quaternion-valued memristor-based neural networks (QVMNN) at the presence of time varying delays. Differential inclusion and fixed time stability theory are used, and new synchronization conditions are formulated to achieve the synchronization of delayed QVMNN within a fixed time based on a Lyapunov function and a suitable controller. The feasibility of the proposed method is shown through numerical simulations.

Keywords: Fixed time synchronization; Lyapunov function; Quaternion; Time varying delays; Memristor-based neural networks
\end{abstract}

\section{Introduction}

Synchronization control is the hot topic of research into nonlinear systems, which is widely applied in various areas. Different synchronization schemes have been presented, including exponential synchronization, complete synchronization, projective synchronization, lag synchronization, and adaptive synchronization [1-5]. It is worthy to point out that these kinds of synchronization strategies considered an infinite time. Therefore, the method which can realize the synchronization within a finite time has important practical application values. The finite time synchronization was first introduced by Kamenkov in [6].

As we all know, neural networks (NN) own numerous specialties, including powerful self-learning and tolerance ability, parallel computing, which have been applied in pattern recognition, signal processing, optimal, and so on. In recent years, the study of its dynamical characteristic, such as finite time stability and synchronization, has gained tremendous attention, and considerable works have been done [7-13].

Finite time synchronization relies on its setting time, which depends on its initial error. To resolve this issue, the concept of fixed time synchronization (FTS) is introduced in [14], implying that the setting time has a convergence upper bound which is independent of the initial values. Because of this merit, FTS has attracted great attention in lots of fields, including power system [15], traffic [16], and consensus [17]. So far, some inter-

(c) The Author(s) 2020. This article is licensed under a Creative Commons Attribution 4.0 International License, which permits use sharing, adaptation, distribution and reproduction in any medium or format, as long as you give appropriate credit to the original author(s) and the source, provide a link to the Creative Commons licence, and indicate if changes were made. The images or other third party material in this article are included in the article's Creative Commons licence, unless indicated otherwise in a credit line to the material. If material is not included in the article's Creative Commons licence and your intended use is not permitted by statutory regulation or exceeds the permitted use, you will need to obtain permission directly from the copyright holder. To view a copy of this licence, visit http://creativecommons.org/licenses/by/4.0/. 
esting results on FTS of NN have been presented [18-24]. Based on Lyapunov functionals, FTS for recurrent memristive NN (MNN) with time delay was presented in [21]. In [22], authors studied the FTS of complex-valued $\mathrm{NN}(\mathrm{CVNN})$ with discontinuous activation functions and parameter uncertainties by decomposing CVNN into two real-valued NN (RVNN). By applying the Lyapunov stability theorem, Ref. [23] considered the FTS of uncertain Cohen-Grossberg NN with time varying delay. In [24], the FTS for a class of impulsive MNN with time varying delay was discussed through designing a suitable controller.

Recently, some scholars introduced quaternion algebra into NN to form quaternionvalued NN (QVNN). Compared with RVNN and CVNN, QVNN can be applied to deal with multidimensional problems, such as image processing, 3-D wind processing, color night vision [25-27]. Moreover, QVNN possess superiority in dealing with optimization and estimation problems and they have widely potential application in engineering field $[28,29]$. Thereupon, it is necessary to consider QVNN. In recent years, a few results on dynamical properties of QVNN, including stability, periodicity, dissipativity, and passivity, have appeared [30-33]. Moreover, the memristor, as a fourth fundamental circuit element, was reported in Nature. The memristor exhibits numerous superiority such as high density, good scalability. Therefore, many scholars constructed the memristor-based neural networks $(\mathrm{MNN})$ by replacing the traditional resistor with memristor. However, there are few results considering FTS for QVNN [34], not mentioning quaternion-valued MNN (QVMNN). On the other hand, due to the finite switching speed of amplifiers, time delays inevitable exist in NN, which may become a key source of instability, oscillation or chaos in the neural system. Furthermore, the delays are time varying in nature. Consequently, it is necessary to consider QVMNN at the presence of time varying delays in our study.

Motivated by the above discussions, this paper focuses on studying the FTS of QVMNN with time varying delays. The three distinctive advantages are listed below. First, taking time delays and memristor into consideration, the model of QVMNN with time varying delays is established, which is more complex and more general. Second, the issue of FTS in delayed QVMNN is discussed. By applying the fixed time control and inequalities skills, some novel conclusions are developed in this paper. Third, the obtained results in this paper can be applied to handle the RVNN, CVNN, and QVNN with or without delays.

Notations. In this paper, $\mathbb{R}, \mathbb{C}, \mathbb{Q}$ refer to the real numbers, complex numbers, and quaternion numbers, respectively. $C\left([-\tau, 0], \mathbb{Q}^{n}\right)$ denotes the continuous functions from $[-\tau, 0]$ to $\mathbb{Q}^{n}$.

\section{Preliminaries}

A quaternion-valued $y$ is described as $y=y^{R}+\imath y^{I}+\jmath y^{J}+\kappa y^{K}$, where $y^{R}, y^{I}, y^{J}, y^{K} \in \mathbb{R}$, the imaginary units $l, J, \kappa$ can satisfy the Hamilton rules:

\begin{tabular}{r|rrrr} 
& 1 & $\imath$ & $\jmath$ & $\kappa$ \\
\hline 1 & 1 & $\iota$ & $J$ & $\kappa$ \\
$\iota$ & $\iota$ & -1 & $\kappa$ & $-\jmath$ \\
$J$ & $\jmath$ & $-\kappa$ & -1 & $\imath$ \\
$\kappa$ & $\kappa$ & $\jmath$ & $-l$ & -1
\end{tabular}


Remark 1 Unlike real value and complex value, the commutative law is not true for quaternion according to Hamilton rules.

The model of delayed QVMNN is described as follows:

$$
\begin{aligned}
\dot{z}_{p}(t)= & -d_{p} z_{p}(t)+\sum_{q=1}^{n} a_{p q}\left(z_{p}(t)\right) f_{q}\left(z_{q}(t)\right) \\
& +\sum_{q=1}^{n} b_{p q}\left(z_{p}(t)\right) g_{q}\left(z_{q}(t-\tau(t))\right) \\
& +l_{p}(t), \quad t \geq 0, p=1,2, \ldots, n,
\end{aligned}
$$

where $z_{p}(t) \in \mathbb{Q}$ denotes the state vector of the $p$ th neuron. $d_{p}>0$ stands for the selffeedback coefficient, $a_{p q}\left(z_{p}(t)\right), b_{p q}\left(z_{p}(t)\right) \in \mathbb{Q}$ are the memristive connection weight matrices, $f_{q}(\cdot) \in \mathbb{Q}$ stands for the neuron activation function. $\tau(t)$ represents time varying delays, $l_{p} \in \mathbb{Q}$ denotes the external input vector. The initial condition is given by $z(s)=\psi(s) \in C\left([-\tau, 0], \mathbb{Q}^{n}\right),-\tau \leq s \leq 0$. System (1) is considered as a drive system.

The memristor connection weights $a_{p q}\left(z_{p}(t)\right)$ and $b_{p q}\left(z_{p}(t)\right)$ satisfy the following conditions:

$$
\begin{aligned}
& a_{p q}\left(z_{p}(t)\right)= \begin{cases}\hat{a}_{p q}, & \left|z_{p}(t)\right| \leq T_{p} ; \\
\grave{a}_{p q}, & \left|z_{p}(t)\right|>T_{p},\end{cases} \\
& b_{p q}\left(z_{p}(t)\right)= \begin{cases}\hat{b}_{p q}, & \left|z_{p}(t)\right| \leq T_{p} ; \\
\grave{b}_{p q}, & \left|z_{p}(t)\right|>T_{p},\end{cases}
\end{aligned}
$$

where $T_{p}>0$ is the switching jumps, $\dot{a}_{p q}, \grave{a}_{p q}, \hat{b}_{p q}, \grave{b}_{p q} \in \mathbb{Q}$ are constants.

Remark 2 The considered system (1) takes into account quaternion values, time-varying delays, and memristors. Compared with [33, 34], the system in $[33,34]$ is just a particular case of this paper.

To carry forward the main results, some necessary assumptions are presented.

Assumption 1 Suppose $z_{p}=z_{p}^{R}+\imath z_{p}^{I}+\jmath z_{p}^{J}+\kappa z_{p}^{K}$, the activation function

$$
f_{q}\left(z_{q}\right)=f_{q}^{R}\left(z_{q}^{R}\right)+\imath f_{q}^{I}\left(z_{q}^{I}\right)+\jmath f_{q}^{J}\left(z_{q}^{J}\right)+\kappa f_{q}^{K}\left(z_{q}^{K}\right) .
$$

Assumption 2 For $p=1,2, \ldots, n, \forall z_{p}^{\mu}, \tilde{z}_{p}^{\mu} \in \mathbb{R}(\mu=R, I, J, K)$, there exist $l_{p}^{\mu}, h_{p}^{\mu}>0$ such that

$$
\begin{aligned}
& \left|f_{p}^{\mu}\left(z_{p}^{\mu}\right)-f_{p}^{\mu}\left(\tilde{z}_{p}^{\mu}\right)\right| \leq l_{p}^{\mu}\left|z_{p}^{\mu}-\tilde{z}_{p}^{\mu}\right|, \\
& \left|g_{p}^{\mu}\left(z_{p}^{\mu}\right)-g_{p}^{\mu}\left(\tilde{z}_{p}^{\mu}\right)\right| \leq h_{p}^{\mu}\left|z_{p}^{\mu}-\tilde{z}_{p}^{\mu}\right| .
\end{aligned}
$$

Assumption $3 \tau(t)$ is differentiable and satisfies $0 \leq \tau(t) \leq \tau$ and $\dot{\tau}(t) \leq \sigma<1$, where $\sigma$ and $\tau$ are constants. 
By employing quaternion multiplication with the non-commutativity, system (1) can be represented as the following four parts:

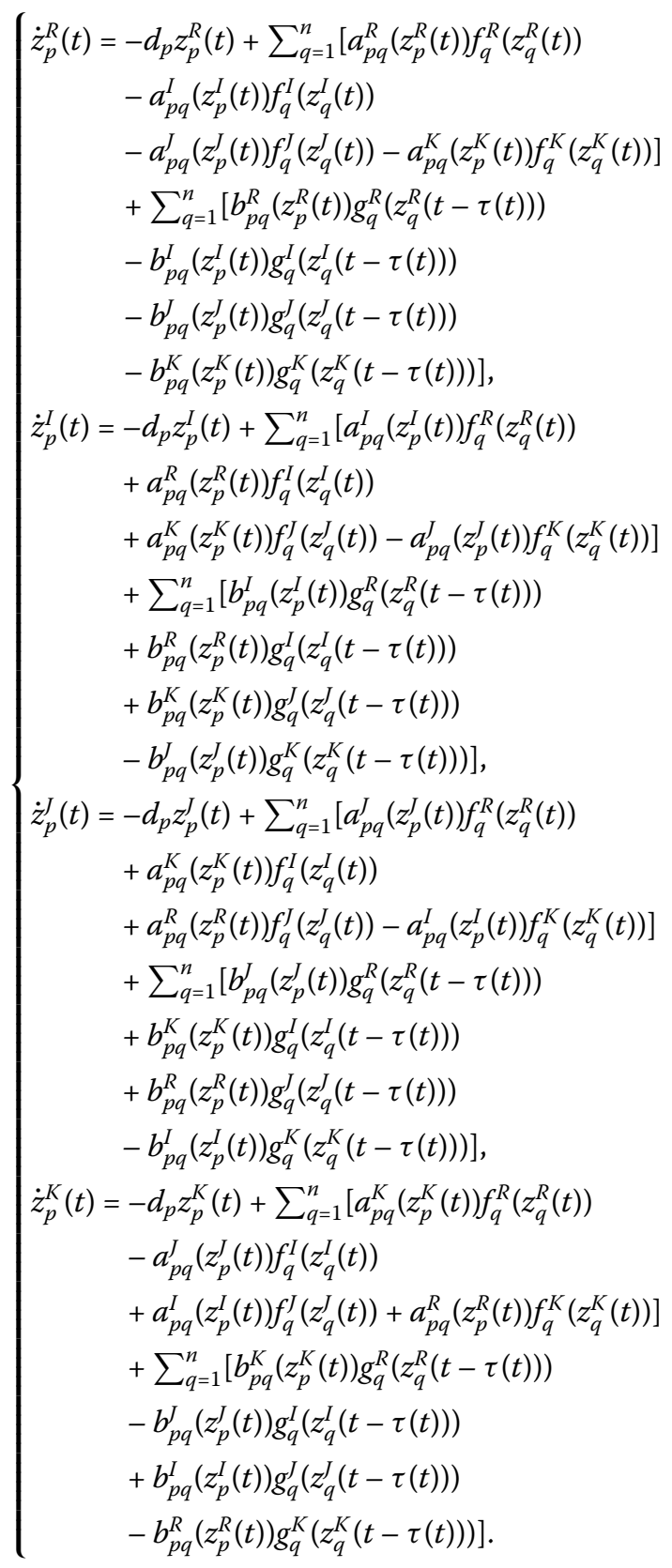

According to the characteristics of memristor and current voltage, we have

$$
\begin{aligned}
& a_{p q}^{\mu}\left(z_{p}^{\mu}(t)\right)= \begin{cases}\hat{a}_{p q}^{\mu}, & \left|z_{p}^{\mu}(t)\right| \leq T_{p} \\
\grave{a}_{p q}^{\mu}, & \left|z_{p}^{\mu}(t)\right|>T_{p}\end{cases} \\
& b_{p q}^{\mu}\left(z_{p}^{\mu}(t)\right)= \begin{cases}\hat{b}_{p q}^{\mu}, & \left|z_{p}^{\mu}(t)\right| \leq T_{p} \\
\dot{b}_{p q}^{\mu}, & \left|z_{p}^{\mu}(t)\right|>T_{p}\end{cases}
\end{aligned}
$$

where $T_{p}>0$ is the switching jumps, $\hat{a}_{p q}^{\mu}, \grave{a}_{p q}^{\mu}, \hat{b}_{p q}^{\mu}, \grave{b}_{p q}^{\mu}$ are constants, $\mu=R, I, J, K$. 
Applying the theory of differential inclusion and the definition of Filippov solution, system (3) can be rewritten as follows:

$$
\begin{aligned}
& \int \dot{z}_{p}^{R}(t) \in-d_{p} z_{p}^{R}(t)+\sum_{q=1}^{n}\left[\operatorname{co}\left\{\bar{a}_{p q}^{R}, \underline{a}_{p q}^{R}\right\} f_{q}^{R}\left(z_{q}^{R}(t)\right)-\operatorname{co}\left\{\bar{a}_{p q}^{I}, \underline{a}_{p q}^{I}\right\} f_{q}^{I}\left(z_{q}^{I}(t)\right)\right. \\
& \left.-\operatorname{co}\left\{\bar{a}_{p q}^{J}, \underline{a}_{p q}^{J}\right\} f_{q}^{J}\left(z_{q}^{J}(t)\right)-\operatorname{co}\left\{\bar{a}_{p q}^{K}, \underline{a}_{p q}^{K}\right\} f_{q}^{K}\left(z_{q}^{K}(t)\right)\right] \\
& +\sum_{q=1}^{n}\left[\operatorname{co}\left\{\bar{b}_{p q}^{R}, \underline{b}_{p q}^{R}\right\} g_{q}^{R}\left(z_{q}^{R}(t-\tau(t))\right)-\operatorname{co}\left\{\bar{b}_{p q}^{I}, \underline{b}_{p q}^{I}\right\} g_{q}^{I}\left(z_{q}^{I}(t-\tau(t))\right)\right. \\
& \left.-\operatorname{co}\left\{\bar{b}_{p q}^{J}, \underline{b}_{p q}^{J}\right\} g_{q}^{J}\left(z_{q}^{J}(t-\tau(t))\right)-\operatorname{co}\left\{\bar{b}_{p q}^{K}, \underline{b}_{p q}^{K}\right\} g_{q}^{K}\left(z_{q}^{K}(t-\tau(t))\right)\right], \\
& \dot{z}_{p}^{I}(t) \in-d_{p} z_{p}^{I}(t)+\sum_{q=1}^{n}\left[\operatorname{co}\left\{\bar{a}_{p q}^{I}, \underline{a}_{p q}^{I}\right\} f_{q}^{R}\left(z_{q}^{R}(t)\right)+\operatorname{co}\left\{\bar{a}_{p q}^{R}, \underline{a}_{p q}^{R}\right\} f_{q}^{I}\left(z_{q}^{I}(t)\right)\right. \\
& \left.+\operatorname{co}\left\{\bar{a}_{p q}^{K}, \underline{a}_{p q}^{K}\right\} f_{q}^{J}\left(z_{q}^{J}(t)\right)-\operatorname{co}\left\{\bar{a}_{p q}^{J}, \underline{a}_{p q}^{J}\right\} f_{q}^{K}\left(z_{q}^{K}(t)\right)\right] \\
& +\sum_{q=1}^{n}\left[\operatorname{co}\left\{\bar{b}_{p q}^{I}, \underline{b}_{p q}^{I}\right\} g_{q}^{R}\left(z_{q}^{R}(t-\tau(t))\right)+\operatorname{co}\left\{\bar{b}_{p q}^{R}, \underline{b}_{p q}^{R}\right\} g_{q}^{I}\left(z_{q}^{I}(t-\tau(t))\right)\right. \\
& \left.+\operatorname{co}\left\{\bar{b}_{p q}^{K}, \underline{b}_{p q}^{K}\right\} g_{q}^{J}\left(z_{q}^{J}(t-\tau(t))\right)-\operatorname{co}\left\{\bar{b}_{p q}^{J}, \underline{b}_{p q}^{J}\right\} g_{q}^{K}\left(z_{q}^{K}(t-\tau(t))\right)\right], \\
& \dot{z}_{p}^{J}(t) \in-d_{p} z_{p}^{J}(t)+\sum_{q=1}^{n}\left[\operatorname{co}\left\{\bar{a}_{p q}^{J}, \underline{a}_{p q}^{J}\right\} f_{q}^{R}\left(z_{q}^{R}(t)\right)+\operatorname{co}\left\{\bar{a}_{p q}^{K}, a_{p q}^{K}\right\} f_{q}^{I}\left(z_{q}^{I}(t)\right)\right. \\
& \left.+\operatorname{co}\left\{\bar{a}_{p q}^{R}, \underline{a}_{p q}^{R}\right\} f_{q}^{J}\left(z_{q}^{J}(t)\right)-\operatorname{co}\left\{\bar{a}_{p q}^{I}, \underline{a}_{p q}^{I}\right\} f_{q}^{K}\left(z_{q}^{K}(t)\right)\right] \\
& +\sum_{q=1}^{n}\left[\operatorname{co}\left\{\bar{b}_{p q}^{J}, \underline{b}_{p q}^{J}\right\} g_{q}^{R}\left(z_{q}^{R}(t-\tau(t))\right)+\operatorname{co}\left\{\bar{b}_{p q}^{K}, \underline{b}_{p q}^{K}\right\} g_{q}^{I}\left(z_{q}^{I}(t-\tau(t))\right)\right. \\
& \left.+\operatorname{co}\left\{\bar{b}_{p q}^{R}, \underline{b}_{p q}^{R}\right\} g_{q}^{J}\left(z_{q}^{J}(t-\tau(t))\right)-\operatorname{co}\left\{\bar{b}_{p q}^{I}, \underline{b}_{p q}^{I}\right\} g_{q}^{K}\left(z_{q}^{K}(t-\tau(t))\right)\right], \\
& \dot{z}_{p}^{K}(t) \in-d_{p} z_{p}^{K}(t)+\sum_{q=1}^{n}\left[c o\left\{\bar{a}_{p q}^{K}, \underline{a}_{p q}^{K}\right\} f_{q}^{R}\left(z_{q}^{R}(t)\right)-\operatorname{co}\left\{\bar{a}_{p q}^{I}, \underline{a}_{p q}^{J}\right\} f_{q}^{I}\left(z_{q}^{I}(t)\right)\right. \\
& \left.+\operatorname{co}\left\{\bar{a}_{p q}^{I}, \underline{a}_{p q}^{I}\right\} f_{q}^{J}\left(z_{q}^{J}(t)\right)+\operatorname{co}\left\{\bar{a}_{p q}^{R}, \underline{a}_{p q}^{R}\right\} f_{q}^{K}\left(z_{q}^{K}(t)\right)\right] \\
& +\sum_{q=1}^{n}\left[\operatorname{co}\left\{\bar{b}_{p q}^{K}, \underline{b}_{p q}^{K}\right\} g_{q}^{R}\left(z_{q}^{R}(t-\tau(t))\right)-\operatorname{co}\left\{\bar{b}_{p q}^{J}, \underline{b}_{p q}^{J}\right\} g_{q}^{I}\left(z_{q}^{I}(t-\tau(t))\right)\right. \\
& \left.+\operatorname{co}\left\{\bar{b}_{p q}^{I}, \underline{b}_{p q}^{I}\right\} g_{q}^{J}\left(z_{q}^{J}(t-\tau(t))\right)-\operatorname{co}\left\{\bar{b}_{p q}^{R}, \underline{b}_{p q}^{R}\right\} g_{q}^{K}\left(z_{q}^{K}(t-\tau(t))\right)\right] \text {. }
\end{aligned}
$$

Or equivalently, there exist $\hat{a}_{p q}^{\mu} \in \operatorname{co}\left\{\bar{a}_{p q}^{\mu}, \underline{a}_{p q}^{\mu}\right\}, \hat{b}_{p q}^{\mu} \in \operatorname{co}\left\{\bar{b}_{p q}^{\mu}, \underline{b}_{p q}^{\mu}\right\}$ such that

$$
\left\{\begin{aligned}
\dot{z}_{p}^{R}(t)= & -d_{p} z_{p}^{R}(t)+\sum_{q=1}^{n}\left[\hat{a}_{p q}^{R} f_{q}^{R}\left(z_{q}^{R}(t)\right)-\hat{a}_{p q}^{I} f_{q}^{I}\left(z_{q}^{I}(t)\right)-\hat{a}_{p q}^{I} f_{q}^{J}\left(z_{q}^{J}(t)\right)\right. \\
& \left.-\hat{a}_{p q}^{K} f_{q}^{K}\left(z_{q}^{K}(t)\right)\right]+\sum_{q=1}^{n}\left[\hat{b}_{p q}^{R} g_{q}^{R}\left(z_{q}^{R}(t-\tau(t))\right)-\hat{b}_{p q}^{I} g_{q}^{I}\left(z_{q}^{I}(t-\tau(t))\right)\right. \\
& \left.-\hat{b}_{p q}^{I} g_{q}^{J}\left(z_{q}^{J}(t-\tau(t))\right)-\hat{b}_{p q}^{K} g_{q}^{K}\left(z_{q}^{K}(t-\tau(t))\right)\right] \\
\dot{z}_{p}^{I}(t)= & -d_{p} z_{p}^{I}(t)+\sum_{q=1}^{n}\left[\hat{a}_{p q}^{I} f_{q}^{R}\left(z_{q}^{R}(t)\right)+\hat{a}_{p q}^{R} f_{q}^{I}\left(z_{q}^{I}(t)\right)+\hat{a}_{p q}^{K} f_{q}^{J}\left(z_{q}^{I}(t)\right)\right. \\
& \left.-\hat{a}_{p q}^{I}\left(z_{p}^{I}(t)\right) f_{q}^{K}\left(z_{q}^{K}(t)\right)\right]+\sum_{q=1}^{n}\left[\hat{b}_{p q}^{I} g_{q}^{R}\left(z_{q}^{R}(t-\tau(t))\right)+\hat{b}_{p q}^{R} g_{q}^{I}\left(z_{q}^{I}(t-\tau(t))\right)\right. \\
& \left.+\hat{b}_{p q}^{K} g_{q}^{J}\left(z_{q}^{I}(t-\tau(t))\right)-\hat{b}_{p q}^{I} g_{q}^{K}\left(z_{q}^{K}(t-\tau(t))\right)\right], \\
\dot{z}_{p}^{I}(t)= & -d_{p} z_{p}^{I}(t)+\sum_{q=1}^{n}\left[\hat{a}_{p q}^{I} f_{q}^{R}\left(z_{q}^{R}(t)\right)+\hat{a}_{p q}^{K} f_{q}^{I}\left(z_{q}^{I}(t)\right)+\hat{a}_{p q}^{R} f_{q}^{J}\left(z_{q}^{I}(t)\right)\right. \\
& \left.-\hat{a}_{p q}^{I} f_{q}^{K}\left(z_{q}^{K}(t)\right)\right]+\sum_{q=1}^{n}\left[\hat{b}_{p q}^{I} g_{q}^{R}\left(z_{q}^{R}(t-\tau(t))\right)+\hat{b}_{p q}^{K} g_{q}^{I}\left(z_{q}^{I}(t-\tau(t))\right)\right. \\
& \left.+\hat{b}_{p q}^{R} g_{q}^{J}\left(z_{q}^{I}(t-\tau(t))\right)-\hat{b}_{p q}^{I} g_{q}^{K}\left(z_{q}^{K}(t-\tau(t))\right)\right] \\
\dot{z}_{p}^{K}(t)= & -d_{p} z_{p}^{K}(t)+\sum_{q=1}^{n}\left[\hat{a}_{p q}^{K} f_{q}^{R}\left(z_{q}^{R}(t)\right)-\hat{a}_{p q}^{I} f_{q}^{I}\left(z_{q}^{I}(t)\right)+\hat{a}_{p q}^{I} f_{q}^{I}\left(z_{q}^{J}(t)\right)\right. \\
& \left.+\hat{a}_{p q}^{R} f_{q}^{K}\left(z_{q}^{K}(t)\right)\right]+\sum_{q=1}^{n}\left[\hat{b}_{p q}^{K} g_{q}^{R}\left(z_{q}^{R}(t-\tau(t))\right)-\hat{b}_{p q}^{I} g_{q}^{I}\left(z_{q}^{I}(t-\tau(t))\right)\right. \\
& \left.+\hat{b}_{p q}^{I} g_{q}^{I}\left(z_{q}^{I}(t-\tau(t))\right)-\hat{b}_{p q}^{R} g_{q}^{K}\left(z_{q}^{K}(t-\tau(t))\right)\right] .
\end{aligned}\right.
$$

Make the definition that $\bar{a}_{p q}^{\mu}=\max \left\{\hat{a}_{p q}^{\mu}, \grave{a}_{p q}^{\mu}\right\}, \underline{a}_{p q}^{\mu}=\min \left\{\hat{a}_{p q}^{\mu}, \grave{a}_{p q}^{\mu}\right\}, \bar{b}_{p q}^{\mu}=\max \left\{\hat{b}_{p q}^{\mu}, \grave{b}_{p q}^{\mu}\right\}, \underline{b}_{p q}^{\mu}=$ $\min \left\{\hat{b}_{p q}^{\mu}, \grave{b}_{p q}^{\mu}\right\}, \mu=R, I, J, K$. 
The response system is given by

$$
\begin{aligned}
\dot{\tilde{z}}_{p}(t)= & -d_{p} \tilde{z}_{p}(t)+\sum_{q=1}^{n} a_{p q}\left(\tilde{z}_{p}(t)\right) f_{q}\left(\tilde{z}_{q}(t)\right) \\
& +\sum_{q=1}^{n} b_{p q}\left(\tilde{z}_{p}(t)\right) g_{q}\left(\tilde{z}_{q}(t-\tau(t))\right) \\
& +l_{p}(t)+u_{p}(t), \quad t \geq 0, p=1,2, \ldots, n,
\end{aligned}
$$

where $u_{p}(t) \in \mathbb{Q}$ is an appropriate controller. The initial condition is $\tilde{z}(s)=\varphi(s) \in$ $C\left([-\tau, 0], \mathbb{Q}^{n}\right),-\tau \leq s \leq 0$.

Similarly, system (7) can be written as follows:

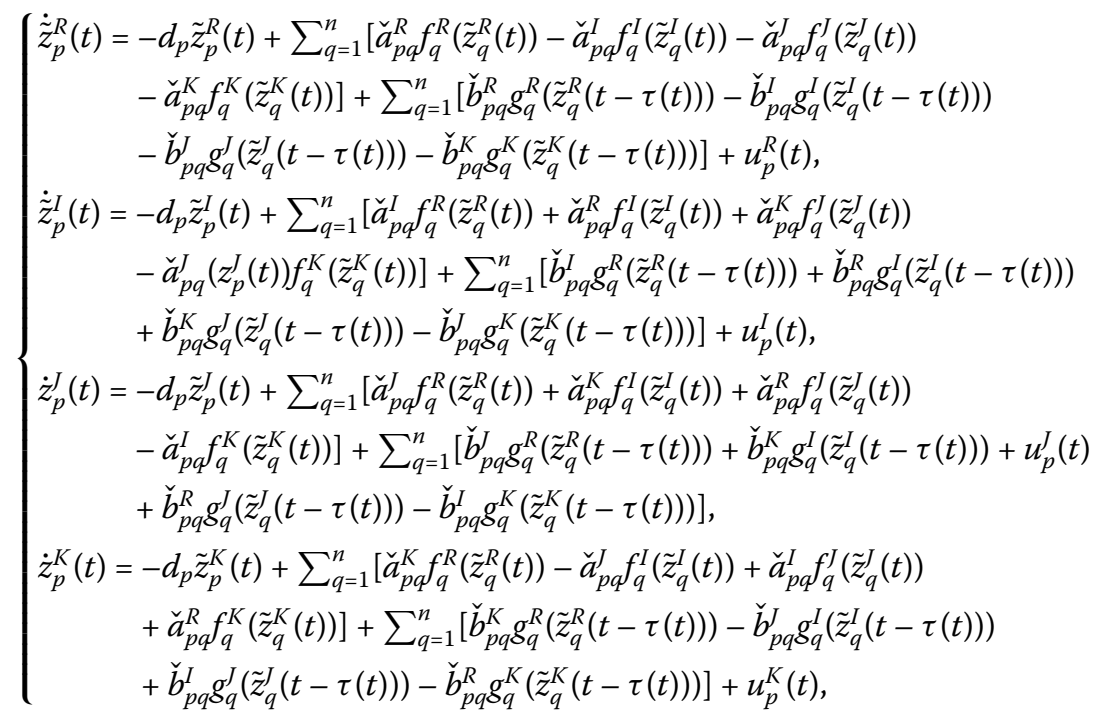

where $\check{a}_{p q}^{\mu} \in \operatorname{co}\left\{\bar{a}_{p q}^{\mu}, \underline{a}_{p q}^{\mu}\right\}, \check{b}_{p q}^{\mu} \in \operatorname{co}\left\{\bar{b}_{p q}^{\mu}, \underline{b}_{p q}^{\mu}\right\}$.

Let $e_{p}(t)=\tilde{z}_{p}(t)-z_{p}(t)$ be the error of synchronization, giving that the system error can be modeled as follows:

$$
\begin{aligned}
\dot{e}_{p}(t) \in & -d_{p} e_{p}(t)+\sum_{q=1}^{n} c o\left\{\bar{a}_{p q}, \underline{a}_{p q}\right\} F_{q}\left(e_{q}(t)\right) \\
& +\sum_{q=1}^{n} c o\left\{\bar{b}_{p q}, \underline{b}_{p q}\right\} G_{q}\left(e_{q}(t-\tau(t))\right) \\
& +u_{p}(t), \quad t \geq 0, p=1,2, \ldots, n,
\end{aligned}
$$

or equivalently, there exist $\bar{a}_{p q} \in \operatorname{co}\left\{\bar{a}_{p q}, \underline{a}_{p q}\right\}, \bar{b}_{p q} \in \operatorname{co}\left\{\bar{b}_{p q}, \underline{b}_{p q}\right\}$ such that

$$
\begin{aligned}
\dot{e}_{p}(t)= & -d_{p} e_{p}(t)+\sum_{q=1}^{n} \bar{a}_{p q} F_{q}\left(e_{q}(t)\right)+\sum_{q=1}^{n} \bar{b}_{p q} G_{q}\left(e_{q}(t-\tau(t))\right) \\
& +u_{p}(t), \quad t \geq 0, p=1,2, \ldots, n,
\end{aligned}
$$


where $F_{q}\left(e_{q}(t)\right)=f_{q}\left(\tilde{z}_{q}(t)\right)-f_{q}\left(z_{q}(t)\right), G_{q}\left(e_{q}\left(t-\tau_{q}\right)\right)=g_{q}\left(\tilde{z}_{q}\left(t-\tau_{q}\right)\right)-g_{q}\left(z_{q}\left(t-\tau_{q}\right)\right)$. The initial condition is $\phi(s)=\psi(s)-\varphi(s)$.

Based on the above analysis, the error system $e_{p}(t) \triangleq e_{p}^{R}(t)+\imath e_{p}^{I}(t)+\jmath e_{p}^{J}(t)+\kappa e_{p}^{K}(t)$ can be represented as the following four parts:

$$
\begin{aligned}
\dot{e}_{p}^{R}(t)= & -d_{p} e_{p}^{R}(t)+\sum_{q=1}^{n}\left[\bar{a}_{p q}^{R} F_{q}^{R}\left(e_{q}^{R}(t)\right)-\bar{a}_{p q}^{I} F_{q}^{I}\left(e_{q}^{I}(t)\right)-\bar{a}_{p q}^{I} F_{q}^{J}\left(e_{q}^{J}(t)\right)\right. \\
& \left.-\bar{a}_{p q}^{K} F_{q}^{K}\left(e_{q}^{K}(t)\right)\right] \\
& +\sum_{q=1}^{n}\left[\bar{b}_{p q}^{R} G_{q}^{R}\left(e_{q}^{R}(t-\tau(t))\right)-\bar{b}_{p q}^{I} G_{q}^{I}\left(e_{q}^{I}(t-\tau(t))\right)-\bar{b}_{p q}^{J} G_{q}^{J}\left(e_{q}^{J}(t-\tau(t))\right)\right. \\
& \left.-\bar{b}_{p q}^{K} G_{q}^{K}\left(e_{q}^{K}(t-\tau(t))\right)\right]+u_{p}^{R}(t), \\
\dot{e}_{p}^{I}(t)= & -d_{p} e_{p}^{I}(t)+\sum_{q=1}^{n}\left[\bar{a}_{p q}^{I} F_{q}^{R}\left(e_{q}^{R}(t)\right)+\bar{a}_{p q}^{R} F_{q}^{I}\left(e_{q}^{I}(t)\right)+\bar{a}_{p q}^{K} F_{q}^{J}\left(e_{q}^{J}(t)\right)\right. \\
& \left.-\bar{a}_{p q}^{I} F_{q}^{K}\left(e_{q}^{K}(t)\right)\right] \\
& +\sum_{q=1}^{n}\left[\bar{b}_{p q}^{I} G_{q}^{R}\left(e_{q}^{R}(t-\tau(t))\right)+\bar{b}_{p q}^{R} G_{q}^{I}\left(e_{q}^{I}(t-\tau(t))\right)+\bar{b}_{p q}^{K} G_{q}^{J}\left(e_{q}^{J}(t-\tau(t))\right)\right. \\
& \left.-\bar{b}_{p q}^{J} G_{q}^{K}\left(e_{q}^{K}(t-\tau(t))\right)\right]+u_{p}^{I}(t), \\
\dot{e}_{p}^{I}(t)= & -d_{p} e_{p}^{J}(t)+\sum_{q=1}^{n}\left[\bar{a}_{p q}^{I} F_{q}^{R}\left(e_{q}^{R}(t)\right)+\bar{a}_{p q}^{K} F_{q}^{I}\left(e_{q}^{I}(t)\right)+\bar{a}_{p q}^{R} F_{q}^{J}\left(e_{q}^{J}(t)\right)\right. \\
& \left.-\bar{a}_{p q}^{I} F_{q}^{K}\left(e_{q}^{K}(t)\right)\right] \\
& +\sum_{q=1}^{n}\left[\bar{b}_{p q}^{I} G_{q}^{R}\left(e_{q}^{R}(t-\tau(t))\right)+\bar{b}_{p q}^{K} G_{q}^{I}\left(e_{q}^{I}(t-\tau(t))\right)+\bar{b}_{p q}^{R} G_{q}^{J}\left(e_{q}^{J}(t-\tau(t))\right)\right. \\
& \left.-\bar{b}_{p q}^{I} G_{q}^{K}\left(e_{q}^{K}(t-\tau(t))\right)\right]+u_{p}^{J}(t), \\
\dot{e}_{p}^{K}(t)= & -d_{p} e_{p}^{K}(t)+\sum_{q=1}^{n}\left[\bar{a}_{p q}^{K} F_{q}^{R}\left(e_{q}^{R}(t)\right)-\bar{a}_{p q}^{I} F_{q}^{I}\left(e_{q}^{I}(t)\right)+\bar{a}_{p q}^{I} F_{q}^{J}\left(e_{q}^{J}(t)\right)\right. \\
& \left.+\bar{a}_{p q}^{R} F_{q}^{K}\left(e_{q}^{K}(t)\right)\right] \\
& +\sum_{q=1}^{n}\left[\bar{b}_{p q}^{K} G_{q}^{R}\left(e_{q}^{R}(t-\tau(t))\right)-\bar{b}_{p q}^{I} G_{q}^{I}\left(e_{q}^{I}(t-\tau(t))\right)+\bar{b}_{p q}^{I} G_{q}^{J}\left(e_{q}^{J}(t-\tau(t))\right)\right. \\
& \left.-\bar{b}_{p q}^{R} G_{q}^{K}\left(e_{q}^{K}(t-\tau(t))\right)\right]+u_{p}^{K}(t),
\end{aligned}
$$

where $F_{q}^{\mu}\left(e_{q}^{\mu}(t)\right)=f_{q}^{\mu}\left(\tilde{z}_{q}^{\mu}(t)\right)-f_{q}^{\mu}\left(z_{q}^{\mu}(t)\right), G_{q}^{\mu}\left(e_{q}^{\mu}\left(t-\tau_{q}\right)\right)=g_{q}^{\mu}\left(\tilde{z}_{q}^{\mu}\left(t-\tau_{q}\right)\right)-g_{q}^{\mu}\left(z_{q}^{\mu}\left(t-\tau_{q}\right)\right), \bar{a}_{p q}^{\mu} \in$ $\operatorname{co}\left\{\bar{a}_{p q}^{\mu}, a_{p q}^{\mu}\right\}, \bar{b}_{p q}^{\mu} \in \operatorname{co}\left\{\bar{b}_{p q}^{\mu}, \underline{b}_{p q}^{\mu}\right\}, \mu=R, I, J, K$.

For the subsequent discussion, the definitions and lemmas are given.

Definition 1 ([6]) For any initial values $\phi$, at an existing time of $0<T(\phi)<\infty$ such that

(1) $\lim _{t \rightarrow T(\phi)}\|e(t, \phi)\|=0$,

(2) $\|e(t, \phi)\|=0, t>T(\phi)$,

drive-response systems (1) and (7) can obtain the finite time synchronization.

Definition 2 Drive-response systems (1) and (7) are said to reach the FTS if the conditions of finite time synchronization hold and the setting time $T(\phi)$ is upper bounded by $T_{\max }$, i.e., $T(\phi) \leq T_{\max }$.

Lemma 1 ([35]) If $V: R^{n} \rightarrow R_{+} \bigcup\{0\}$ is a continuous radially unbounded function satisfying:

(1) $V(y)=0 \Leftrightarrow y=0$

(2) For $\xi, \eta>0, \delta>1,0<\theta<1$, the following inequality holds:

$$
D^{+} V(y(t)) \leq-\xi V^{\delta}(y(t))-\eta V^{\theta}(y(t)), \quad \forall y(t)
$$


then the origin is fixed time stable; moreover,

$$
V(y(t)) \equiv 0, \quad t \geq T\left(y_{0}\right)
$$

with the setting time bounded by

$$
T\left(y_{0}\right) \leq T_{\max }:=\frac{1}{\eta}\left(\frac{\eta}{\xi}\right)^{\frac{1-\theta}{\delta-\theta}}\left(\frac{1}{1-\theta}+\frac{1}{\delta-1}\right) .
$$

Lemma 2 ([36]) Assume $y_{1}, y_{2}, \ldots, y_{n} \geq 0,0<\varepsilon \leq 1, \zeta>1$, then the following inequalities hold:

$$
\sum_{q=1}^{n} y_{p}^{\varepsilon} \geq\left(\sum_{q=1}^{n} y_{p}\right)^{\varepsilon}, \quad \sum_{q=1}^{n} y_{p}^{\zeta} \geq n^{1-\zeta}\left(\sum_{q=1}^{n} y_{p}\right)^{\zeta} .
$$

\section{Fixed time synchronization}

In order to achieve the FTS of delayed QVMNN, the controller is designed as follows:

$$
\left\{\begin{array}{l}
u_{p}^{R}(t)=-\lambda_{1 p}^{R} e_{p}^{R}(t)-\operatorname{sign}\left(e_{p}^{R}(t)\right)\left[\lambda_{2 p}^{R}\left|e_{p}^{R}(t)\right|^{\delta}+\lambda_{3 p}^{R}\left|e_{p}^{R}(t)\right|^{\theta}+\lambda_{4 p}^{R}\left|e_{p}^{R}(t-\tau(t))\right|\right], \\
u_{p}^{I}(t)=-\lambda_{1 p}^{I} e_{p}^{I}(t)-\operatorname{sign}\left(e_{p}^{I}(t)\right)\left[\lambda_{2 p}^{I}\left|e_{p}^{I}(t)\right|^{\delta}+\lambda_{3 p}^{I}\left|e_{p}^{I}(t)\right|^{\theta}+\lambda_{4 p}^{I}\left|e_{p}^{I}(t-\tau(t))\right|\right], \\
u_{p}^{J}(t)=-\lambda_{1 p}^{J} e_{p}^{J}(t)-\operatorname{sign}\left(e_{p}^{J}(t)\right)\left[\lambda_{2 p}^{J}\left|e_{p}^{J}(t)\right|^{\delta}+\lambda_{3 p}^{J}\left|e_{p}^{J}(t)\right|^{\theta}+\lambda_{4 p}^{J}\left|e_{p}^{J}(t-\tau(t))\right|\right], \\
u_{p}^{K}(t)=-\lambda_{1 p}^{K} e_{p}^{K}(t)-\operatorname{sign}\left(e_{p}^{K}(t)\right)\left[\lambda_{2 p}^{K}\left|e_{p}^{K}(t)\right|^{\delta}+\lambda_{3 p}^{K}\left|e_{p}^{K}(t)\right|^{\theta}+\lambda_{4 p}^{K}\left|e_{p}^{K}(t-\tau(t))\right|\right],
\end{array}\right.
$$

where $\lambda_{1 p}^{\mu}, \lambda_{2 p}^{\mu}, \lambda_{3 p}^{\mu}, \lambda_{4 p}^{\mu}(\mu=R, I, J, K, p=1,2, \ldots, n)$ are control gains, $\delta>1,0<\theta<1$.

Theorem 1 Let Assumptions 1-3 hold, when the control gains satisfy the following conditions:

$$
\left\{\begin{array}{l}
\lambda_{1 p}^{\mu} \geq \sum_{q=1}^{n}\left[\left|\bar{a}_{q p}^{R}\right|+\left|\bar{a}_{q p}^{I}\right|+\left|\bar{a}_{q p}^{J}\right|+\left|\bar{a}_{q p}^{K}\right|\right] l_{p}^{\mu}-d_{p}, \\
\lambda_{4 p}^{\mu} \geq \sum_{q=1}^{n}\left[\left|\bar{b}_{q p}^{R}\right|+\left|\bar{b}_{q p}^{I}\right|+\left|\bar{b}_{q p}^{J}\right|+\left|\bar{b}_{q p}^{K}\right|\right] h_{p}^{\mu}, \\
\lambda_{2 p}^{\mu} \geq 0, \quad \lambda_{3 p}^{\mu} \geq 0, \quad p=1,2, \ldots, n, \mu=R, I, J, K,
\end{array}\right.
$$

where $\delta>1,0<\theta<1$, then the response system (7) can synchronize the drive system (1) in a fixed time under controller (12). The fixed time $T$ is estimated as follows:

$$
T \leq \frac{1}{\lambda_{3}}\left(\frac{\lambda_{3}}{\lambda_{2}(4 n)^{1-\delta}}\right)^{\frac{1-\theta}{\delta-\theta}}\left(\frac{1}{1-\theta}+\frac{1}{\delta-1}\right)
$$

where $\lambda_{2}=\min _{p}\left\{\lambda_{2 p}^{\mu}\right\}, \lambda_{3}=\min _{p}\left\{\lambda_{3 p}^{\mu}\right\}$.

Proof Constructing the Lyapunov function

$$
\begin{aligned}
V(t) & =V_{1}(t)+V_{2}+V_{3}(t)(t)+V_{4}(t) \\
& \triangleq \sum_{p=1}^{n}\left|e_{p}^{R}(t)\right|+\sum_{p=1}^{n}\left|e_{p}^{I}(t)\right|+\sum_{p=1}^{n}\left|e_{p}^{J}(t)\right|+\sum_{p=1}^{n}\left|e_{p}^{K}(t)\right|,
\end{aligned}
$$


taking the time derivative of $V_{1}(t)$, one gets

$$
\begin{aligned}
\dot{V}_{1}(t)= & \sum_{p=1}^{n} \operatorname{sign}\left(e_{p}^{R}(t)\right)\left\{-d_{p} e_{p}^{R}(t)+\sum_{q=1}^{n}\left[\bar{a}_{p q}^{R} F_{q}^{R}\left(e_{q}^{R}(t)\right)-\bar{a}_{p q}^{I} F_{q}^{I}\left(e_{q}^{I}(t)\right)-\bar{a}_{p q}^{J} F_{q}^{J}\left(e_{q}^{J}(t)\right)\right.\right. \\
& \left.-\bar{a}_{p q}^{K} F_{q}^{K}\left(e_{q}^{K}(t)\right)\right]+\sum_{q=1}^{n}\left[\bar{b}_{p q}^{R} G_{q}^{R}\left(e_{q}^{R}(t-\tau(t))\right)-\bar{b}_{p q}^{I} G_{q}^{I}\left(e_{q}^{I}(t-\tau(t))\right)\right. \\
& \left.-\bar{b}_{p q}^{I} G_{q}^{J}\left(e_{q}^{J}(t-\tau(t))\right)-\bar{b}_{p q}^{K} G_{q}^{K}\left(e_{q}^{K}(t-\tau(t))\right)\right] \\
& \left.-\lambda_{1 p}^{R} e_{p}^{R}(t)-\operatorname{sign}\left(e_{p}^{R}(t)\right)\left[\lambda_{2 p}^{R}\left|e_{p}^{R}(t)\right|^{\delta}+\lambda_{3 p}^{R}\left|e_{p}^{R}(t)\right|^{\theta}+\lambda_{4 p}^{R}\left|e_{p}^{R}(t-\tau(t))\right|\right]\right\} \\
\leq & \sum_{p=1}^{n}\left\{-\left(d_{p}+\lambda_{1 p}^{R}\right) \mid e_{p}^{R}(t)\right) \mid+\sum_{q=1}^{n}\left[\left|\bar{a}_{p q}^{R}\right| l_{q}^{R}\left|e_{q}^{R}(t)\right|+\left|\bar{a}_{p q}^{I}\right| l_{q}^{I}\left|e_{q}^{I}(t)\right|+\left|\bar{a}_{p q}^{I}\right| l_{q}^{J}\left|e_{q}^{J}(t)\right|\right. \\
& \left.+\left|\bar{a}_{p q}^{K}\right| l_{q}^{K}\left|e_{q}^{K}(t)\right|\right]+\sum_{q=1}^{n}\left[\left|\bar{b}_{p q}^{R}\right| h_{q}^{R}\left|e_{q}^{R}(t-\tau(t))\right|\right. \\
& \left.+\left|\bar{b}_{p q}^{I}\right| h_{q}^{I}\left|e_{q}^{I}(t-\tau(t))\right|+\left|\bar{b}_{p q}^{I}\right| h_{q}^{J}\left|e_{q}^{J}(t-\tau(t))\right|+\left|\bar{b}_{p q}^{K}\right| h_{q}^{K}\left|e_{q}^{K}(t-\tau(t))\right|\right] \\
& \left.-\lambda_{2 p}^{R}\left|e_{p}^{R}(t)\right|^{\delta}-\lambda_{3 p}^{R}\left|e_{p}^{R}(t)\right|^{\theta}-\lambda_{4 p}^{R}\left|e_{p}^{R}(t-\tau(t))\right|\right\} \\
= & \left.\sum_{p=1}^{n}\left\{-d_{p}-\lambda_{1 p}^{R}+\sum_{q=1}^{n}\left|\bar{a}_{q p}^{R}\right| l_{p}^{R}\right\} \mid e_{p}^{R}(t)\right) \mid+\sum_{p=1}^{n} \sum_{q=1}^{n}\left[\left|\bar{a}_{p q}^{I}\right| l_{q}^{I}\left|e_{q}^{I}(t)\right|+\left|\bar{a}_{p q}^{I}\right| l_{q}^{J}\left|e_{q}^{J}(t)\right|\right. \\
& \left.+\left|\bar{a}_{p q}^{K}\right| l l_{q}^{K}\left|e_{q}^{K}(t)\right|\right]+\sum_{p=1}^{n}\left\{-\lambda_{4 p}^{R}+\sum_{q=1}^{n}\left|\bar{b}_{q p}^{R}\right| h_{p}^{R}\right\}\left|e_{p}^{R}(t-\tau(t))\right| \\
& +\sum_{p=1}^{n} \sum_{q=1}^{n}\left[\left|\bar{b}_{p q}^{I}\right| h_{q}^{I}\left|e_{q}^{I}(t-\tau(t))\right|+\left|\bar{b}_{p q}^{J}\right| h_{q}^{J}\left|e_{q}^{J}(t-\tau(t))\right|+\left|\bar{b}_{p q}^{K}\right| h_{q}^{K}\left|e_{q}^{K}(t-\tau(t))\right|\right] \\
& -\sum_{p=1}^{n}\left[\lambda_{2 p}^{R}\left|e_{p}^{R}(t)\right|^{\delta}+\lambda_{3 p}^{R}\left|e_{p}^{R}(t)\right|^{\theta}\right] . \\
& \\
&
\end{aligned}
$$

Similarly:

$$
\begin{aligned}
\dot{V}_{2}(t) \leq & \sum_{p=1}^{n}\left\{-d_{p}-\lambda_{1 p}^{I}+\sum_{q=1}^{n}\left|\bar{a}_{q p}^{R}\right| l_{p}^{I}\right\}\left|e_{p}^{I}(t)\right|+\sum_{p=1}^{n} \sum_{q=1}^{n}\left[\left|\bar{a}_{p q}^{I}\right| l_{q}^{R}\left|e_{q}^{R}(t)\right|+\left|\bar{a}_{p q}^{K}\right| l_{q}^{J}\left|e_{q}^{J}(t)\right|\right. \\
& \left.+\left|\bar{a}_{p q}^{I}\right| l_{q}^{K}\left|e_{q}^{K}(t)\right|\right]+\sum_{p=1}^{n}\left\{-\lambda_{4 p}^{I}+\sum_{q=1}^{n}\left|\bar{b}_{q p}^{R}\right| h_{p}^{I}\right\}\left|e_{p}^{I}(t-\tau(t))\right| \\
& +\sum_{p=1}^{n} \sum_{q=1}^{n}\left[\left|\bar{b}_{p q}^{I}\right| h_{q}^{R}\left|e_{q}^{R}(t-\tau(t))\right|+\left|\bar{b}_{p q}^{K}\right| h_{q}^{J}\left|e_{q}^{J}(t-\tau(t))\right|+\left|\bar{b}_{p q}^{J}\right| h_{q}^{K}\left|e_{q}^{K}(t-\tau(t))\right|\right] \\
& -\sum_{p=1}^{n}\left[\lambda_{2 p}^{I}\left|e_{p}^{I}(t)\right|^{\delta}+\lambda_{3 p}^{I}\left|e_{p}^{I}(t)\right|^{\theta}\right], \\
\dot{V}_{3}(t) \leq & \sum_{p=1}^{n}\left\{-d_{p}-\lambda_{1 p}^{J}+\sum_{q=1}^{n}\left|\bar{a}_{q p}^{R}\right| l_{p}^{J}\right\}\left|e_{p}^{J}(t)\right|+\sum_{p=1}^{n} \sum_{q=1}^{n}\left[\left|\bar{a}_{p q}^{J}\right| l_{q}^{R}\left|e_{q}^{R}(t)\right|+\left|\bar{a}_{p q}^{K}\right| l_{q}^{I}\left|e_{q}^{I}(t)\right|\right.
\end{aligned}
$$




$$
\begin{aligned}
& \left.+\left|\bar{a}_{p q}^{I}\right| l_{q}^{K}\left|e_{q}^{K}(t)\right|\right]+\sum_{p=1}^{n}\left\{-\lambda_{4 p}^{J}+\sum_{q=1}^{n}\left|\bar{b}_{q p}^{R}\right| h_{p}^{J}\right\}\left|e_{p}^{J}(t-\tau(t))\right| \\
& +\sum_{p=1}^{n} \sum_{q=1}^{n}\left[\left|\bar{b}_{p q}^{J}\right| h_{q}^{R}\left|e_{q}^{R}(t-\tau(t))\right|+\left|\bar{b}_{p q}^{K}\right| h_{q}^{I}\left|e_{q}^{I}(t-\tau(t))\right|+\left|\bar{b}_{p q}^{I}\right| h_{q}^{K}\left|e_{q}^{K}(t-\tau(t))\right|\right] \\
& -\sum_{p=1}^{n}\left[\lambda_{2 p}^{J}\left|e_{p}^{J}(t)\right|^{\delta}+\lambda_{3 p}^{J}\left|e_{p}^{J}(t)\right|^{\theta}\right], \\
\dot{V}_{4}(t) \leq & \sum_{p=1}^{n}\left\{-d_{p}-\lambda_{1 p}^{K}+\sum_{q=1}^{n}\left|\bar{a}_{q p}^{R}\right| l_{p}^{K}\right\}\left|e_{p}^{K}(t)\right|+\sum_{p=1}^{n} \sum_{q=1}^{n}\left[\left|\bar{a}_{p q}^{K}\right| l_{q}^{R}\left|e_{q}^{R}(t)\right|+\left|\bar{a}_{p q}^{J}\right| l_{q}^{I}\left|e_{q}^{I}(t)\right|\right. \\
& \left.+\left|\bar{a}_{p q}^{I}\right| l_{q}^{J}\left|e_{q}^{J}(t)\right|\right]+\sum_{p=1}^{n}\left\{-\lambda_{4 p}^{K}+\sum_{q=1}^{n}\left|\bar{b}_{q p}^{R}\right| h_{p}^{K}\right\}\left|e_{p}^{K}(t-\tau(t))\right| \\
& +\sum_{p=1}^{n} \sum_{q=1}^{n}\left[\left|\bar{b}_{p q}^{K}\right| h_{q}^{R}\left|e_{q}^{R}(t-\tau(t))\right|+\left|\bar{b}_{p q}^{J}\right| h_{q}^{I}\left|e_{q}^{I}(t-\tau(t))\right|+\left|\bar{b}_{p q}^{I}\right| h_{q}^{J}\left|e_{q}^{J}(t-\tau(t))\right|\right] \\
& -\sum_{p=1}^{n}\left[\lambda_{2 p}^{K}\left|e_{p}^{K}(t)\right|^{\delta}+\lambda_{3 p}^{K}\left|e_{p}^{K}(t)\right|^{\theta}\right] .
\end{aligned}
$$

By combining the above inequalities and according to Lemma 2, one gets the following:

$$
\begin{aligned}
& \left.\dot{V}(t) \leq \sum_{p=1}^{n}\left\{-d_{p}-\lambda_{1 p}^{R}+\sum_{q=1}^{n}\left[\left|\bar{a}_{q p}^{R}\right|+\left|\bar{a}_{q p}^{I}\right|+\left|\bar{a}_{q p}^{I}\right|+\left|\bar{a}_{q p}^{K}\right|\right] l_{p}^{R}\right\} \mid e_{p}^{R}(t)\right) \mid \\
& \left.+\sum_{p=1}^{n}\left\{-d_{p}-\lambda_{1 p}^{I}+\sum_{q=1}^{n}\left[\left|\bar{a}_{q p}^{R}\right|+\left|\bar{a}_{q p}^{I}\right|+\left|\bar{a}_{q p}^{I}\right|+\left|\bar{a}_{q p}^{K}\right|\right] l_{p}^{I}\right\} \mid e_{p}^{I}(t)\right) \mid \\
& \left.+\sum_{p=1}^{n}\left\{-d_{p}-\lambda_{1 p}^{J}+\sum_{q=1}^{n}\left[\left|\bar{a}_{q p}^{R}\right|+\left|\bar{a}_{q p}^{I}\right|+\left|\bar{a}_{q p}^{I}\right|+\left|\bar{a}_{q p}^{K}\right|\right] l_{p}^{J}\right\} \mid e_{p}^{J}(t)\right) \mid \\
& \left.+\sum_{p=1}^{n}\left\{-d_{p}-\lambda_{1 p}^{K}+\sum_{q=1}^{n}\left[\left|\bar{a}_{q p}^{R}\right|+\left|\bar{a}_{q p}^{I}\right|+\left|\bar{a}_{q p}^{I}\right|+\left|\bar{a}_{q p}^{K}\right|\right] l_{p}^{K}\right\} \mid e_{p}^{K}(t)\right) \mid \\
& +\sum_{p=1}^{n}\left\{-\lambda_{4 p}^{R}+\sum_{q=1}^{n}\left[\left|\bar{b}_{q p}^{R}\right|+\left|\bar{b}_{q p}^{I}\right|+\left|\bar{b}_{q p}^{J}\right|+\left|\bar{b}_{q p}^{K}\right|\right] h_{p}^{R}\right\}\left|e_{p}^{R}(t-\tau(t))\right| \\
& +\sum_{p=1}^{n}\left\{-\lambda_{4 p}^{I}+\sum_{q=1}^{n}\left[\left|\bar{b}_{q p}^{R}\right|+\left|\bar{b}_{q p}^{I}\right|+\left|\bar{b}_{q p}^{I}\right|+\left|\bar{b}_{q p}^{K}\right|\right] h_{p}^{I}\right\}\left|e_{p}^{I}(t-\tau(t))\right| \\
& +\sum_{p=1}^{n}\left\{-\lambda_{4 p}^{J}+\sum_{q=1}^{n}\left[\left|\bar{b}_{q p}^{R}\right|+\left|\bar{b}_{q p}^{I}\right|+\left|\bar{b}_{q p}^{J}\right|+\left|\bar{b}_{q p}^{K}\right|\right] h_{p}^{J}\right\}\left|e_{p}^{J}(t-\tau(t))\right| \\
& +\sum_{p=1}^{n}\left\{-\lambda_{4 p}^{K}+\sum_{q=1}^{n}\left[\left|\bar{b}_{q p}^{R}\right|+\left|\bar{b}_{q p}^{I}\right|+\left|\bar{b}_{q p}^{I}\right|+\left|\bar{b}_{q p}^{K}\right|\right] h_{p}^{K}\right\}\left|e_{p}^{K}(t-\tau(t))\right| \\
& -\sum_{p=1}^{n}\left[\lambda_{2 p}^{R}\left|e_{p}^{R}(t)\right|^{\delta}+\lambda_{3 p}^{R}\left|e_{p}^{R}(t)\right|^{\theta}\right]-\sum_{p=1}^{n}\left[\lambda_{2 p}^{I}\left|e_{p}^{I}(t)\right|^{\delta}+\lambda_{3 p}^{I}\left|e_{p}^{I}(t)\right|^{\theta}\right]
\end{aligned}
$$




$$
\begin{aligned}
& -\sum_{p=1}^{n}\left[\lambda_{2 p}^{J}\left|e_{p}^{J}(t)\right|^{\delta}+\lambda_{3 p}^{J}\left|e_{p}^{J}(t)\right|^{\theta}\right]-\sum_{p=1}^{n}\left[\lambda_{2 p}^{K}\left|e_{p}^{K}(t)\right|^{\delta}+\lambda_{3 p}^{K}\left|e_{p}^{K}(t)\right|^{\theta}\right] \\
\leq & -\sum_{p=1}^{n}\left[\lambda_{2 p}^{R}\left|e_{p}^{R}(t)\right|^{\delta}+\lambda_{3 p}^{R}\left|e_{p}^{R}(t)\right|^{\theta}\right]-\sum_{p=1}^{n}\left[\lambda_{2 p}^{I}\left|e_{p}^{I}(t)\right|^{\delta}+\lambda_{3 p}^{I}\left|e_{p}^{I}(t)\right|^{\theta}\right] \\
& -\sum_{p=1}^{n}\left[\lambda_{2 p}^{J}\left|e_{p}^{J}(t)\right|^{\delta}+\lambda_{3 p}^{J}\left|e_{p}^{J}(t)\right|^{\theta}\right]-\sum_{p=1}^{n}\left[\lambda_{2 p}^{K}\left|e_{p}^{K}(t)\right|^{\delta}+\lambda_{3 p}^{K}\left|e_{p}^{K}(t)\right|^{\theta}\right] \\
\leq & -\lambda_{2} \sum_{p=1}^{n}\left[\left|e_{p}^{R}(t)\right|^{\delta}+\left|e_{p}^{I}(t)\right|^{\delta}+\left|e_{p}^{J}(t)\right|^{\delta}+\left|e_{p}^{K}(t)\right|^{\delta}\right] \\
& -\lambda_{3} \sum_{p=1}^{n}\left[\left|e_{p}^{R}(t)\right|^{\theta}+\left|e_{p}^{I}(t)\right|^{\theta}+\left|e_{p}^{J}(t)\right|^{\theta}+\left|e_{p}^{K}(t)\right|^{\theta}\right] \\
\leq & -\lambda_{2}(4 n)^{1-\delta}\left[\sum_{p=1}^{n}\left|e_{p}^{R}(t)\right|+\left|e_{p}^{I}(t)\right|+\left|e_{p}^{J}(t)\right|+\left|e_{p}^{K}(t)\right|\right]^{\delta} \\
& -\lambda_{3}\left[\sum_{p=1}^{n}\left|e_{p}^{R}(t)\right|+\left|e_{p}^{I}(t)\right|+\left|e_{p}^{J}(t)\right|+\left|e_{p}^{K}(t)\right|\right]^{\theta} \\
= & -\lambda_{2}(4 n)^{1-\delta} V^{\delta}(t)-\lambda_{3} V^{\theta}(t) .
\end{aligned}
$$

Therefore, following from Lemma 1, the FTS between drive-response systems (1) and (7) can be achieved. Moreover, the upper bound of setting time is estimated by $T \leq$ $\frac{1}{\lambda_{3}}\left(\frac{\lambda_{3}}{\lambda_{2}(4 n)^{1-\delta}}\right)^{\frac{1-\theta}{\delta-\theta}}\left(\frac{1}{1-\theta}+\frac{1}{\delta-1}\right)$.

Remark 3 Ref. [34] investigated the FTS of QVNN without considering time-delay, and some conditions of FTS and bound of the settling time were obtained. In our paper, the FTS of more complex QVMNN at the presence of time varying delays is considered. Moreover, the design of controllers and the conclusions are more complex and more general than those in Ref. [34].

Remark 4 Recently, many excellent results on FTS of RVNN and CVNN [18-24] have been presented. Compared with previous works, QVNN possess superiority in dealing with multidimensional problems, and they have widely potential application in engineering field. Hence, our result is more general and meaningful.

Remark 5 It is worthy of pointing out that quaternion multiplication does not satisfy the commutative rule. Therefore, traditional methods and techniques for solving problems of CVNN or RVNN cannot be directly employed to study QVNN. To avoid the non-commutativity of quaternion multiplication, a feasible method is to decompose the quaternion-valued systems into real-valued systems. Choosing different approaches on investigating the dynamics characteristics of QVNN is still an open and challenging task. In the future, we are focusing on studying the synchronization of QVMNN via direct method. 


\section{Numerical example}

The following QVMNN with time delay is taken as a drive system:

$$
\begin{aligned}
\dot{z}_{p}(t)= & -d_{p} z_{p}(t)+\sum_{q=1}^{2} a_{p q}\left(z_{p}(t)\right) f_{q}\left(z_{q}(t)\right) \\
& +\sum_{q=1}^{2} b_{p q}\left(z_{p}(t)\right) g_{q}\left(z_{q}(t-\tau(t))\right) \\
& +l_{p}(t), \quad t \geq 0, p=1,2,
\end{aligned}
$$

where $z_{p}(t)=z_{p}^{R}(t)+l z_{p}^{I}(t)+\jmath z_{p}^{J}(t)+\kappa z_{p}^{K}(t), \tau(t)=0.2+0.78 \sin (t) . l_{1}(t)=0.5+0.4 l-0.5 J-$ $0.3 \kappa, l_{2}(t)=-0.3+0.3 l+2 \jmath-1 \kappa, d_{1}=d_{2}=1$.

$$
\begin{aligned}
& a_{11}^{R}\left(x_{1}^{R}\right)=\left\{\begin{array}{ll}
-0.4, & \left|x_{1}^{R}\right| \leq 1, \\
0.1, & \left|x_{1}^{R}\right|>1,
\end{array} \quad a_{12}^{R}\left(x_{1}^{R}\right)= \begin{cases}0.1, & \left|x_{1}^{R}\right| \leq 1, \\
-0.1, & \left|x_{1}^{R}\right|>1,\end{cases} \right. \\
& a_{21}^{R}\left(x_{2}^{R}\right)=\left\{\begin{array}{ll}
-0.1, & \left|x_{2}^{R}\right| \leq 1, \\
0.1, & \left|x_{2}^{R}\right|>1,
\end{array} \quad a_{22}^{R}\left(x_{2}^{R}\right)= \begin{cases}0.1, & \left|x_{2}^{R}\right| \leq 1, \\
-0.1, & \left|x_{2}^{R}\right|>1,\end{cases} \right. \\
& a_{11}^{I}\left(x_{1}^{I}\right)=\left\{\begin{array}{ll}
0.2, & \left|x_{1}^{I}\right| \leq 1, \\
0.1, & \left|x_{1}^{I}\right|>1,
\end{array} \quad a_{12}^{I}\left(x_{1}^{I}\right)= \begin{cases}0.1, & \left|x_{1}^{I}\right| \leq 1, \\
-0.1, & \left|x_{1}^{I}\right|>1,\end{cases} \right. \\
& a_{21}^{I}\left(x_{2}^{I}\right)=\left\{\begin{array}{ll}
0.2, & \left|x_{2}^{I}\right| \leq 1, \\
0.1, & \left|x_{2}^{I}\right|>1,
\end{array} \quad a_{22}^{I}\left(x_{2}^{I}\right)= \begin{cases}0.1, & \left|x_{2}^{I}\right| \leq 1, \\
-0.1, & \left|x_{2}^{I}\right|>1,\end{cases} \right. \\
& a_{11}^{J}\left(x_{1}^{J}\right)=\left\{\begin{array}{ll}
-0.1, & \left|x_{1}^{J}\right| \leq 1, \\
0.1, & \left|x_{1}^{J}\right|>1,
\end{array} \quad a_{12}^{J}\left(x_{1}^{J}\right)= \begin{cases}0.1, & \left|x_{1}^{J}\right| \leq 1, \\
-0.1, & \left|x_{1}^{J}\right|>1,\end{cases} \right. \\
& a_{21}^{J}\left(x_{2}^{J}\right)=\left\{\begin{array}{ll}
-0.1, & \left|x_{2}^{J}\right| \leq 1, \\
0.2, & \left|x_{2}^{J}\right|>1,
\end{array} \quad a_{22}^{J}\left(x_{2}^{J}\right)= \begin{cases}0.1, & \left|x_{2}^{J}\right| \leq 1, \\
-0.1, & \left|x_{2}^{J}\right|>1,\end{cases} \right. \\
& a_{11}^{K}\left(x_{1}^{K}\right)=\left\{\begin{array}{ll}
-0.1, & \left|x_{1}^{K}\right| \leq 1, \\
0.2, & \left|x_{1}^{K}\right|>1,
\end{array} \quad a_{12}^{K}\left(x_{1}^{K}\right)= \begin{cases}0.1, & \left|x_{1}^{K}\right| \leq 1, \\
-0.1, & \left|x_{1}^{K}\right|>1,\end{cases} \right. \\
& a_{21}^{K}\left(x_{2}^{K}\right)=\left\{\begin{array}{ll}
-0.1, & \left|x_{2}^{K}\right| \leq 1, \\
0.1, & \left|x_{2}^{K}\right|>1,
\end{array} \quad a_{22}^{K}\left(x_{2}^{K}\right)= \begin{cases}0.1, & \left|x_{2}^{K}\right| \leq 1, \\
0.2, & \left|x_{2}^{K}\right|>1,\end{cases} \right. \\
& b_{11}^{R}\left(x_{1}^{R}\right)=\left\{\begin{array}{ll}
0.2, & \left|x_{1}^{R}\right| \leq 1, \\
0.1, & \left|x_{1}^{R}\right|>1,
\end{array} \quad b_{12}^{R}\left(x_{1}^{R}\right)= \begin{cases}0.1, & \left|x_{1}^{R}\right| \leq 1, \\
-0.1, & \left|x_{1}^{R}\right|>1,\end{cases} \right. \\
& b_{21}^{R}\left(x_{2}^{R}\right)=\left\{\begin{array}{ll}
0.2, & \left|x_{2}^{R}\right| \leq 1, \\
0.1, & \left|x_{2}^{R}\right|>1,
\end{array} \quad b_{22}^{R}\left(x_{2}^{R}\right)= \begin{cases}0.1, & \left|x_{2}^{R}\right| \leq 1, \\
-0.1, & \left|x_{2}^{R}\right|>1,\end{cases} \right. \\
& b_{11}^{I}\left(x_{1}^{I}\right)=\left\{\begin{array}{ll}
-0.1, & \left|x_{1}^{I}\right| \leq 1, \\
0.1, & \left|x_{1}^{I}\right|>1,
\end{array} \quad b_{12}^{I}\left(x_{1}^{I}\right)= \begin{cases}0.1, & \left|x_{1}^{I}\right| \leq 1, \\
0.2, & \left|x_{1}^{I}\right|>1,\end{cases} \right.
\end{aligned}
$$




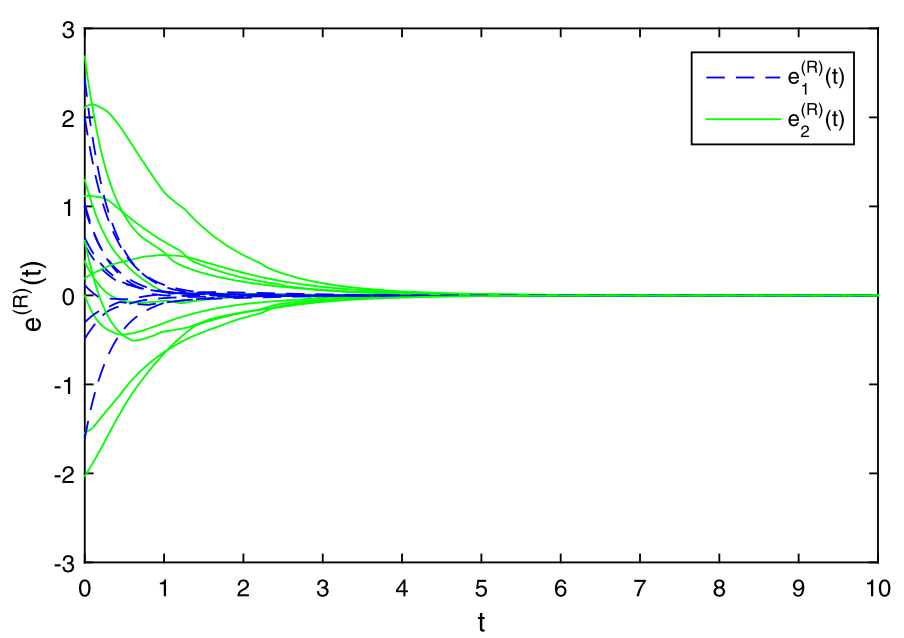

Figure 1 The error curves of $e_{1}^{R}, e_{2}^{R}$

$$
\begin{aligned}
& b_{21}^{I}\left(x_{2}^{I}\right)=\left\{\begin{array}{ll}
-0.1, & \left|x_{2}^{I}\right| \leq 1, \\
0.4, & \left|x_{2}^{I}\right|>1,
\end{array} \quad b_{22}^{I}\left(x_{2}^{I}\right)= \begin{cases}0.1, & \left|x_{2}^{I}\right| \leq 1, \\
0.2, & \left|x_{2}^{I}\right|>1,\end{cases} \right. \\
& b_{11}^{J}\left(x_{1}^{J}\right)=\left\{\begin{array}{ll}
-0.1, & \left|x_{1}^{J}\right| \leq 1, \\
0.2, & \left|x_{1}^{J}\right|>1,
\end{array} \quad b_{12}^{J}\left(x_{1}^{J}\right)= \begin{cases}0.1, & \left|x_{1}^{J}\right| \leq 1, \\
-0.1, & \left|x_{1}^{J}\right|>1,\end{cases} \right. \\
& b_{21}^{J}\left(x_{2}^{J}\right)=\left\{\begin{array}{ll}
-0.1, & \left|x_{2}^{J}\right| \leq 1, \\
0.2, & \left|x_{2}^{J}\right|>1,
\end{array} \quad b_{22}^{J}\left(x_{2}^{J}\right)= \begin{cases}0.1, & \left|x_{2}^{J}\right| \leq 1, \\
-0.1, & \left|x_{2}^{J}\right|>1,\end{cases} \right. \\
& b_{11}^{K}\left(x_{1}^{K}\right)=\left\{\begin{array}{ll}
-0.1, & \left|x_{1}^{K}\right| \leq 1, \\
0.1, & \left|x_{1}^{K}\right|>1,
\end{array} \quad b_{12}^{K}\left(x_{1}^{K}\right)= \begin{cases}0.1, & \left|x_{1}^{K}\right| \leq 1, \\
0.2, & \left|x_{1}^{K}\right|>1,\end{cases} \right. \\
& b_{21}^{K}\left(x_{2}^{K}\right)=\left\{\begin{array}{ll}
-0.1, & \left|x_{2}^{K}\right| \leq 1, \\
0.1, & \left|x_{2}^{K}\right|>1,
\end{array} \quad b_{22}^{K}\left(x_{2}^{K}\right)= \begin{cases}0.1, & \left|x_{2}^{K}\right| \leq 1, \\
0.2, & \left|x_{2}^{K}\right|>1 .\end{cases} \right.
\end{aligned}
$$

The neuron activation function is

$$
f_{p}\left(z_{p}(t)\right)=g_{p}\left(z_{p}(t)\right) \frac{1}{1+e^{z_{p}^{R}(t)}}+\frac{1}{1+e^{z_{p}^{I}(t)}} l+\frac{1}{1+e^{z_{p}}(t)} J+\frac{1}{1+e^{z_{p}^{K}(t)}} \kappa
$$

for $p=1,2$, which implies $l_{p}^{\mu}=h_{p}^{\mu}=1, \mu=R, I, J, K$.

The response system is described as follows:

$$
\begin{aligned}
\dot{\tilde{z}}_{p}(t)= & -d_{p} \tilde{z}_{p}(t)+\sum_{q=1}^{n} a_{p q}\left(\tilde{z}_{p}(t)\right) f_{q}\left(\tilde{z}_{q}(t)\right) \\
& +\sum_{q=1}^{n} b_{p q}\left(\tilde{z}_{p}(t)\right) g_{q}\left(\tilde{z}_{q}\left(t-\tau_{q}\right)\right) \\
& +l_{p}(t)+u_{p}(t), \quad t \geq 0, p=1,2
\end{aligned}
$$




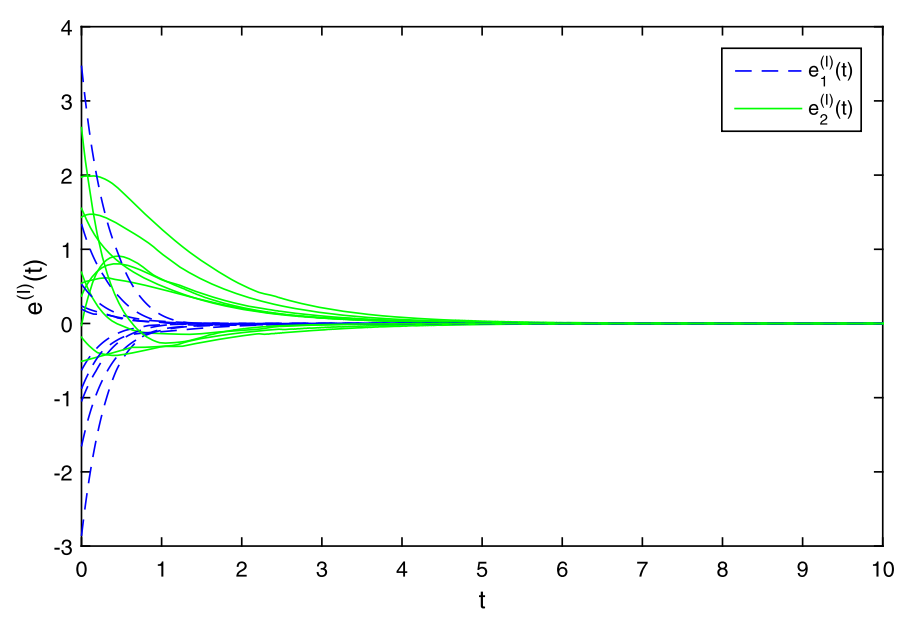

Figure 2 The error curves of $e_{1}^{\prime}, e_{2}^{l}$

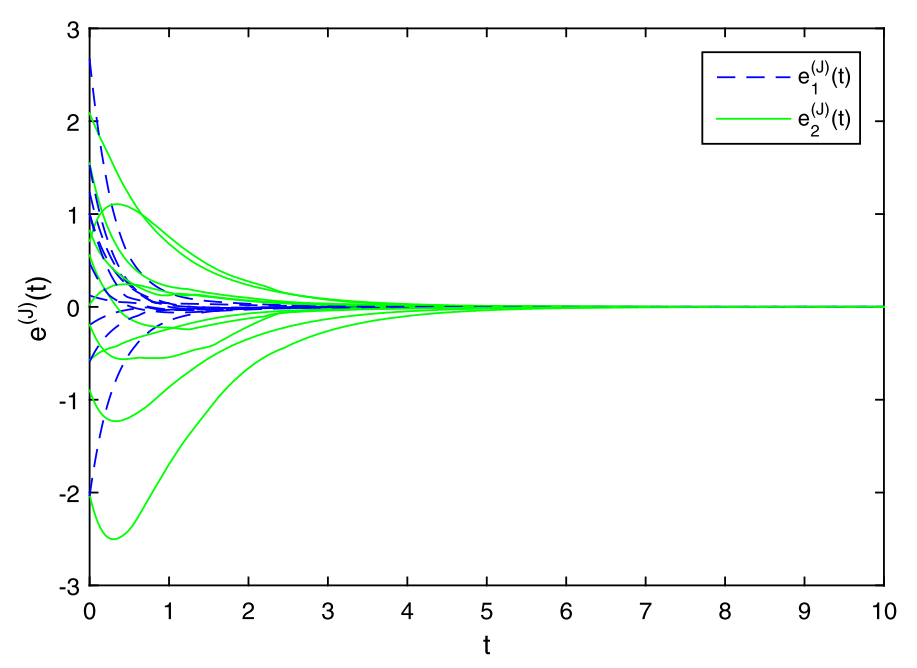

Figure 3 The error curves of $e_{1}^{\jmath}, e_{2}^{J}$

where $\tilde{z}_{p}(t)=\tilde{z}_{p}^{R}(t)+\imath \tilde{z}_{p}^{I}(t)+\jmath \tilde{z}_{p}^{J}(t)+\kappa \tilde{z}_{p}^{K}(t) \cdot u_{p}(t)=u_{p}^{R}(t)+\imath u_{p}^{I}(t)+\jmath u_{p}^{J}(t)+\kappa u_{p}^{K}(t)$ is the controller. The parameters used here are similar to those used in (16). Choosing $\lambda_{1 p}^{\mu}=4$, $\lambda_{2 p}^{\mu}=5, \lambda_{3 p}^{\mu}=10, \lambda_{4 p}^{\mu}=2(\mu=R, I, J, K, p=1,2, \ldots, n), \delta=1.5, \theta=0.5$, the conditions in Theorem 1 hold. According to Theorem 1, drive-response systems (16) and (17) can synchronize with the setting time $T_{\max }=0.6$. Figures $1-4$ show the synchronization trajectories with ten random initial values.

\section{Conclusions}

The FTS issue for a class of QVMNN at the presence of time varying delays is investigated based on fixed time stability theory. With the help of a Lyapunov function and a nonlinear controller, some sufficient conditions are established to implement the FTS of delayed QVMNN. Finally, a numerical example is used to present the effectiveness of the proposed 


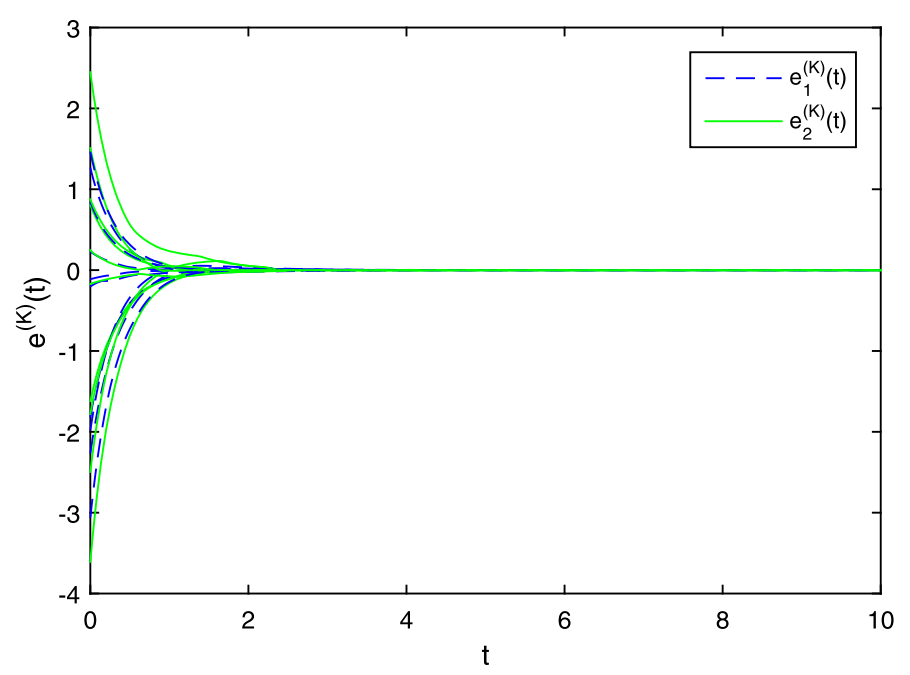

Figure 4 The error curves of $e_{1}^{K}, e_{2}^{K}$

method. The synchronization of delayed QVMNN with parameter uncertainties warrants further investigation.

\section{Acknowledgements}

The authors thank the editor and the anonymous reviewers for their insightful comments and suggestions that improved the quality and presentation of this paper.

\section{Funding}

This study is supported by the National Natural Science Foundation of China (No. 61573096), the Natural Science Foundation of Anhui Province (No. 1908085MA01), the Excellent Young Talents Fund Program of Higher Education Institutions of Anhui Province (No. KJ2018A0365, No. KJ2019A0573, No. KJ2019A0556), and the Special Foundation for Young Scientists of Anhui Province (No. gxyq2019048).

Availability of data and materials

The data used to support the findings of this study are available from the corresponding author upon request.

\section{Competing interests}

The authors declare that they have no competing interests.

\section{Authors' contributions}

All authors contributed equally to the manuscript. All the authors read and approved the final manuscript.

\section{Author details}

${ }^{1}$ School of Mathematics and Physics, Anqing Normal University, Anqing, China. ${ }^{2}$ School of Mathematics, Southeast University, Nanjing, China. ${ }^{3}$ Department of Applied Mathematics, Changsha University of Science and Technology, Changsha, China.

\section{Publisher's Note}

Springer Nature remains neutral with regard to jurisdictional claims in published maps and institutional affiliations.

Received: 9 August 2019 Accepted: 21 February 2020 Published online: 28 February 2020

\section{References}

1. Zhang, W.W., Zhang, H., Cao, J.D., Alsaadi, F.E., Chen, D.Y.: Synchronization in uncertain fractional-order memristive complex-valued neural networks with multiple time delays. Neural Netw. 110, 186-198 (2019)

2. Zhang, L.Z., Yang, Y.Q., Wang, F., Sui, X.: Lag synchronization for fractional-order memristive neural networks with time delay via switching jumps mismatch. J. Franklin Inst. 355, 1217-1240 (2018)

3. Zhang, W.W., Cao, J.D., Wu, R.C., Chen, D.Y., Alsaadi, F.E.: Novel results on projective synchronization of fractional-order neural networks with multiple time delays. Chaos Solitons Fractals 117, 76-83 (2018)

4. Bao, H.B., Park, J.H., Cao, J.D.: Adaptive synchronization of fractional-order memristor-based neural networks with time delay. Nonlinear Dyn. 82, 1343-1354 (2015) 
5. Zhang, G.D., Shen, Y.: Exponential synchronization of delayed memristor-based chaotic neural networks via periodically intermittent control. Neural Netw. 55, 1-10 (2014)

6. Kamenkov, G.: On stability of motion over a finite interval of time. J. Appl. Math. Mech. 17, 529-540 (1953)

7. Zavala-Rio, A., Fantoni, l.: Global finite-time stability characterized through a local notion of homogeneity. IEEE Trans, Autom. Control 59, 471-477 (2014)

8. Yang, H., Jiang, B., Zhao, J.: On finite-time stability of cyclic switched nonlinear systems. IEEE Trans. Autom. Control 60 2201-2206 (2015)

9. Rakkiyappan, R., Velmurugan, G., Cao, J.: Finite-time stability analysis of fractional-order complex-valued memristor-based neural networks with time delays. Nonlinear Dyn. 78, 2823-2836 (2014)

10. Chen, C., Li, L.X., Peng, H.P., Yang, Y.X., Li, T.: Finite-time synchronization of memristor-based neural networks with mixed delays. Neurocomputing 235, 83-89 (2017)

11. Liu, M., Jiang, H.J., Hu, C.: Finite-time synchronization of memristor-based Cohen-Grossberg neural networks with time-varying delays. Neurocomputing 194, 1-9 (2016)

12. Duan, L., Wei, H., Huang, L.H.: Finite-time synchronization of delayed fuzzy cellular neural networks with discontinuous activations. Fuzzy Sets Syst. 361, 56-70 (2019)

13. Wang, L., Song, Q.K.: Pricing policies for dual-channel supply chain with green investment and sales effort under uncertain demand. Math. Comput. Simul. 361, 56-70 (2019)

14. Polyakov, A.: Nonlinear feedback design for fixed-time stabilization of linear control systems. IEEE Trans. Autom. Control 57, 2106-2110 (2012)

15. Ni, J.K., Liu, L., Liu, C.X., Hu, X.Y., Li, S.L.: Fast fixed-time nonsingular terminal sliding mode control and its application to chaos suppression in power system. IEEE Trans. Circuits Syst. II, Express Briefs 64, 151-155 (2017)

16. Muralidharan, A., Pedarsani, R., Varaiya, P.: Analysis of fixed-time control. Transp. Res., Part B, Methodol. 73, 81-90 (2015)

17. Hua, C.C., Li, Y.F., Guan, X.P.: Finite/fixed-time stabilization for nonlinear interconnected systems with dead-zone input. IEEE Trans. Autom. Control 62, 2554-2560 (2017)

18. Liu, X.W., Chen, T.P.: Finite-time and fixed-time cluster synchronization with or without pinning control. IEEE Trans. Cybern. 48, 240-252 (2018)

19. Wang, L.M., Zeng, Z.G., Hu, J.H., Wang, X.P.: Controller design for global fixed-time synchronization of delayed neural networks with discontinuous activations. Neural Netw. 87, 122-131 (2017)

20. Huang, Y.L., Qiu, S.H., Ren, S.Y., Zheng, Z.W.: Fixed-time synchronization of coupled Cohen-Grossberg neural networks with and without parameter uncertainties. Neurocomputing 315, 157-168 (2018)

21. Cao, J.D., Li, R.X.: Fixed-time synchronization of delayed memristor-based recurrent neural networks. Sci. China Inf. Sci. 60, $032201(2017)$

22. Ding, X.S., Cao, J.D., Alsaedi, A., Alsaadi, F.E., Hayat, T.: Robust fixed-time synchronization for uncertain complex-valued neural networks with discontinuous activation functions. Neural Netw. 90, 42-55 (2017)

23. Wan, Y., Cao, J.D., Wen, G.H., Yu, W.W.: Robust fixed-time synchronization of delayed Cohen-Grossberg neural networks. Neural Netw. 73, 86-94 (2016)

24. Zhang, Y.L., Zhuang, J.S., Xia, Y.H., Bai, Y.Z., Cao, J.D., Gu, L.F.: Fixed-time synchronization of the impulsive memristor-based neural networks. Commun. Nonlinear Sci. Numer. Simul. 77, 40-53 (2019)

25. Took, C.C., Mandic, D.P.: The quaternion LMS algorithm for adaptive filtering of hypercomplex processes. IEEE Trans. Signal Process. 57, 1316-1327 (2009)

26. Zou, C.M., Kou, K.I., Wang, Y.L.: Quaternion collaborative and sparse representation with application to color face recognition. IEEE Trans. Signal Process. 25, 3287-3302 (2016)

27. Matsui, N., Isokawa, T., Kusamichi, H., Peper, F., Nishimura, H.: Quaternion neural network with geometrical operators. J. Intell. Fuzzy Syst. 15, 149-164 (2004)

28. Qin, S.T., Feng, J.Q., Song, J.H., Wen, X.N., Xu, C.: A one-layer recurrent neural network for constrained complex-variable convex optimization. IEEE Trans. Neural Netw. Learn. Syst. 29, 534-544 (2018)

29. Sahoo, A., Xu, H., Jagannathan, S.: Neural network-based event-triggered state feedback control of nonlinear continuous-time systems. IEEE Trans. Neural Netw. Learn. Syst. 27, 497-509 (2016)

30. Tu, Z.W., Zhao, Y.X., Ding, N., Feng, Y.M., Zhang, W.: Stability analysis of quaternion-valued neural networks with both discrete and distributed delays. Appl. Math. Comput. 343, 342-353 (2019)

31. Song, Q.K., Chen, X.F.: Multistability analysis of quaternion-valued neural networks with time delays. IEEE Trans. Neural Netw. Learn. Syst. 29, 5430-5440 (2018)

32. Popa, C.A., Kaslik, E.: Multistability and multiperiodicity in impulsive hybrid quaternion-valued neural networks with mixed delays. Neural Netw. 99, 1-18 (2018)

33. Tu, Z.W., Cao, J.D., Alsaedi, A., Hayat, T.: Global dissipativity analysis for delayed quaternion-valued neural networks. Neural Netw. 89, 97-104 (2017)

34. Deng, H., Bao, H.B.: Fixed-time synchronization of quaternion-valued neural networks. Physica A 527, 121351 (2019)

35. Hu, C., Yu, J., Chen, Z.H., Jiang, H.J., Huang, T.W.: Fixed-time stability of dynamical systems and fixed-time synchronization of coupled discontinuous neural networks. Neural Netw. 89, 74-83 (2017)

36. Kanter, I., Kinzel, W., Kanter, E.: Secure exchange of information by synchronization of neural networks. Europhys. Lett. 57, 141-147(2002) 